\title{
A therapeutic conundrum: recurrent cystic-fibrosis-related haemoptysis complicated by acute pulmonary embolism
}

\author{
William G Flight, ${ }^{1,2}$ Rowland J Bright-Thomas, ${ }^{1,2}$ Stephen Butterfield, ${ }^{3}$ \\ Andrew M Jones, ${ }^{1,2}$ A Kevin Webb ${ }^{1,2}$
}

${ }^{1}$ Manchester Adult Cystic Fibrosis Centre, Manchester, UK ${ }^{2}$ Respiratory Research Group, University of Manchester, Manchester, UK

${ }^{3}$ Department of Radiology, University Hospital of South Manchester, Manchester, UK

\section{Correspondence to}

Dr William G Flight, Manchester Adult Cystic Fibrosis Centre, University Hospital of South Manchester NHS Foundation Trust, Southmoor Road, Manchester M23 9LT, UK; william.flight@uhsm.nhs.uk

Received 17 April 2012 Accepted 4 May 2012

Published Online First 7 June 2012

\section{ABSTRACT}

The authors present the case of an older patient with cystic fibrosis (CF) and recurrent haemoptysis complicated by acute pulmonary embolism. The patient was treated successfully with a combination of anticoagulation and bronchial artery embolisation. The management of CF-related haemoptysis, the impact of an ageing CF population and the incidence of thromboembolic disease in CF are discussed.

\section{CASE REPORT}

Dr Flight (WGF): A 72-year-old man with cystic fibrosis (CF) was admitted to hospital with recurrent haemoptysis. He was a compound heterozygote for the F508del and R117H-7T mutations of the cystic fibrosis transmembrane conductance regulator (CFTR) gene. CF had been diagnosed at the age of 65 prior to which he had been labelled as having asthma and non-CF bronchiectasis. Baseline forced expiratory volume in $1 \mathrm{~s}$ was $36 \%$ predicted and he was chronically infected with Pseudomonas aeruginosa and Burkholderia multivorans. His regular therapy included long-term oral corticosteroids for allergic bronchopulmonary aspergillosis and nebulised colistin. In addition to multiple complications of CF he also had a history of cataracts, age-related macular degeneration, hypertension, peptic ulcer disease, colonic polyps, benign prostatic hypertrophy and basal cell carcinomas of the skin.

On the day of admission he had expectorated approximately a third of a cup of bright red blood. Intravenous tranexamic acid was administered at a dose of $1 \mathrm{~g}$ four times daily alongside intravenous temocillin, tobramycin and co-trimoxazole. The haemoptysis gradually resolved and he was discharged 2 weeks later to continue oral tranexamic acid $1 \mathrm{~g}$ twice daily.

Professor Webb (AKW): First, this case illustrates an increasingly important phenomenon in the care of patients with CF: the ageing patient. The survival of people with CF has steadily increased over recent decades ${ }^{1}$ and patients are frequently living into their sixth decade and beyond. Much of this improved life expectancy is due to multidisciplinary specialist care but a proportion is accounted for by greater numbers of late diagnoses afforded by identification of rarer cystic fibrosis transmembrane conductance regulator mutations. Compound heterozygosity for the F508del and R117H-7T mutations has a recognised association with delayed diagnosis of $\mathrm{CF}^{2}$ As patients with CF start to live into old age, CF physicians will increasingly need to be alert to diseases of ageing never previously considered relevant to $\mathrm{CF}$.

Haemoptysis is a common complication of CF with a 5 -year incidence of $9.1 \%$ in one study. ${ }^{3}$ The pathophysiology of haemoptysis in CF is poorly understood but characteristically involves the development of tortuous, dilated vessels in the bronchial circulation. Treatment has been the subject of consensus guidance ${ }^{4}$ and centres on appropriate antibiotic therapy with bronchial artery embolisation (BAE) recommended as the treatment of choice for massive haemoptysis. Tranexamic acid is an anti-fibrinolytic drug that is effective in reducing bleeding related to postpartum haemorrhage and several surgical procedures. Although tranexamic acid is commonly used in CF-related haemoptysis there is no robust evidence to support its efficacy or safety in this setting. Concern has previously been raised over an increased risk of venous thromboembolism with tranexamic acid but this has not been resolved conclusively as yet.

WGF: Following discharge, the patient continued to experience streaks of blood in his sputum on a daily basis. He was re-admitted 2 weeks later with haemoptysis and symptoms of a viral upper respiratory tract infection. On the day of admission he had expectorated half a cupful of fresh red blood. On arrival to hospital he was comfortable at rest with normal vital signs. Oxygen saturations were $97 \%$ on air. An ECG revealed normal sinus rhythm and a chest radiograph was unchanged from previously.

Two hours after presentation, he suddenly developed breathlessness and chest tightness. Examination revealed him to be tachypnoeic, tachycardic and hypotensive. Oxygen saturations had dropped to $78 \%$ on air. A repeat ECG showed new right bundle branch block. An urgent CTpulmonary angiogram demonstrated a large thrombus involving the left main, left upper and left lower lobe pulmonary arteries (see figure 1A). Serum troponin I was elevated at $0.51 \mu \mathrm{g} /$ litre.

AKW: The patient has developed two potentially fatal pulmonary emergencies: persistent major haemoptysis and concurrent acute pulmonary embolism (PE). This scenario is a genuine therapeutic conundrum. Anticoagulation is required to prevent further thrombosis while, simultaneously, 
Figure 1 (A) CT pulmonary angiogram demonstrating thrombus in the left main and upper lobe pulmonary arteries (white arrow). (B) Coronal CT reconstruction illustrating a dilated bronchial artery arising from the descending aorta (black arrow).
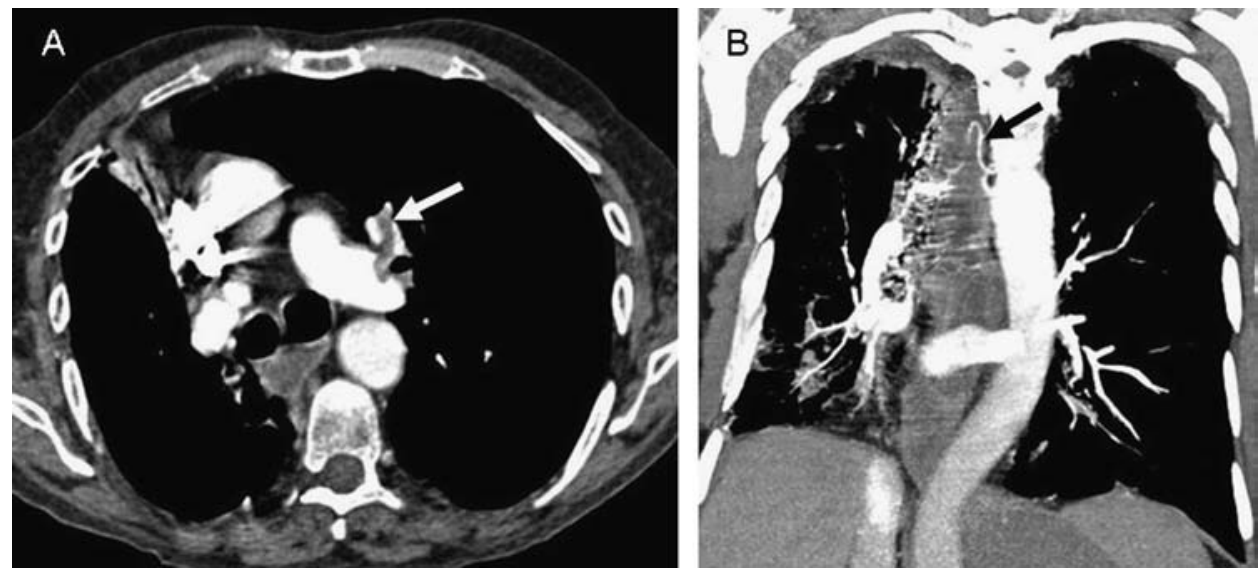

further bleeding must be avoided. When there is a risk of significant haemorrhage, anticoagulation is best achieved with intravenous unfractionated heparin and close monitoring of the activated partial thromboplastin time. In the event of lifethreatening bleeding, the intravenous heparin can be quickly discontinued. If possible, the cause of the bleeding should be identified and reversed. In the event of bleeding preventing safe anticoagulation, the insertion of an inferior vena cava filter is a further therapeutic option.

WGF: Intravenous heparin was commenced and the patient was also treated with intravenous temocillin and tobramycin. High-flow oxygen was administered to achieve target saturations of $94-98 \%$. Fluid resuscitation was given and tranexamic acid was discontinued. An inferior vena cava filter was considered but was not pursued as the CT examination showed no evidence of venous thrombosis in the legs or pelvis. Despite the above measures, the patient experienced ongoing substantial haemoptysis. Advice from the interventional radiology service was sought.

Dr Butterfield (Interventional Radiology): My first step was to review the original CT pulmonary angiogram study. CT reconstructions were performed which revealed a dilated bronchial artery arising from the descending aorta (see figure 1B). We elected to perform a bronchial artery angiogram with the aim of embolising any potential sources of bleeding from the bronchial circulation. The dilated bronchial artery identified on the CT scan was supra-selectively catheterised and embolised to stasis with polyvinyl alcohol.

The available evidence suggests that BAE is an effective treatment for massive haemoptysis in CF. There have been no randomised trials of BAE in CF but large case series suggest that acute bleeding is controlled in up to $97 \%$ of cases with a longterm recurrence rate of $27-46 \%{ }^{5}$ Chest pain is the most common side effect following BAE, although potential complications include an acute neurological deficit due to embolisation of a spinal artery or passage of embolic material through collateral vessels to the cerebral circulation. In the present case the patient experienced mild chest pain for $24 \mathrm{~h}$ after the procedure but did not suffer any more serious adverse events.

WGF: Following BAE the patient's haemoptysis settled completely. Intravenous heparin was converted to low molecular weight heparin and oral warfarin was commenced $48 \mathrm{~h}$ after undergoing BAE. He was discharged at day 16, by which time his symptoms had returned to baseline and he had remained free from haemoptysis.

AKW: The development of PE in a patient with ongoing major bleeding presents a highly challenging clinical situation that may be encountered in a wide variety of medical and surgical specialties. The principles of management in this situation are applicable to a number of conditions in respiratory medicine, such as the patient with lung cancer, aspergilloma or myriad other causes of haemoptysis.

The scenario described is highly unusual in CF, however, and to my knowledge has not been reported before. Idiopathic PE alone is considered exceptionally rare in patients with CF. Previous reports of $\mathrm{PE}$ in $\mathrm{CF}$ have all been associated with venous access device related thrombosis. In this case it is certainly possible that the use of the tranexamic acid contributed to the development of venous thrombosis. The risks associated with such pro-thrombotic drugs remain uncertain and need to be addressed in future studies.

As the CF population ages it is reasonable to expect that the incidence of venous thrombosis in this population will steadily rise. Since haemoptysis is such a common feature of CF, it stands to reason that we shall also see concurrent haemoptysis and thromboembolic disease with increasing frequency.

The key learning points to be taken from this case are:

1. The CF population is getting older and diseases associated with ageing are increasingly relevant to the CF physician.

2. Bronchial artery embolisation represents the therapy of choice for massive haemoptysis in CF.

3. CF physicians should be alert to the possibility of PE in their patients, a diagnosis which is easily overlooked in the context of severe CF lung disease.

4. The conundrum of acute PE in the context of major bleeding requires a delicate balance of anticoagulation and correction of the underlying haemorrhage.

Contributors WGF wrote the first draft. All authors contributed to the final manuscript.

\section{Competing interests None.}

Patient consent Obtained.

Provenance and peer review Not commissioned; internally peer reviewed.

\section{REFERENCES}

1. Dodge J, Lewis $\mathrm{P}$, Stanton $\mathrm{M}$, et al. Cystic fibrosis mortality and survival in the UK: 1947-2003. Eur Respir J 2007;29:522-6.

2. Peckham D, Conway SP, Morton A, et al. Delayed diagnosis of cystic fibrosis associated with $\mathrm{R} 117 \mathrm{H}$ on a background of $7 \mathrm{~T}$ polythymidine tract at intron 8. J Cyst Fibros 2006;5:63-5.

3. Efrati 0, Harash 0, Rivlin J, et al. Hemoptysis in Israeli CF patients-prevalence, treatment, and clinical characteristics. J Cyst Fibros 2008;7:301-6.

4. Flume PA, Mogayzel PJ, Robinson KA, et al. Cystic fibrosis pulmonary guidelines: pulmonary complications: hemoptysis and pneumothorax. Am J Respir Crit Care Med 2010;182:298-306.

5. Vidal V, Therasse $E$, Berthiaume $Y$, et al. Bronchial artery embolization in adults with cystic fibrosis: impact on the clinical course and survival. J Vasc Interv Radiol 2006;17:953-8 